\title{
Practical Authentication and Access Control for Software-Defined Networking over Optical Networks*
}

\author{
Joo Yeon Cho \\ ADVA Optical Networking SE \\ Fraunhoferstrasse 9a \\ Martinsried, Germany 82152 \\ JCho@advaoptical.com
}

\author{
Thomas Szyrkowiec \\ ADVA Optical Networking SE \\ Fraunhoferstrasse 9a \\ Martinsried, Germany 82152 \\ TSzyrkowiec@advaoptical.com
}

\begin{abstract}
A framework of Software-Defined Networking (SDN) provides a centralized and integrated method to manage and control modern optical networks. Unfortunately, the centralized and programmable structure of SDN introduces several new security threats, which may allow an adversary to take over the entire operation of the network. In this paper, we investigate the potential security threats of SDN over optical networks and propose a mutual authentication and a fine-grained access control mechanism, which are essential to avoid an unauthorized access to the network. The proposed schemes are based only on cryptographic hash functions and do not require an installation of the complicated cryptographic library such as SSL. Unlike conventional authentication and access control schemes, the proposed schemes are flexible, compact and, in addition, are resistant to quantum computer attacks, which may become critical in the near future.
\end{abstract}

\section{CCS CONCEPTS}

.Security and privacy $\rightarrow$ Network security; Security protocols;

\section{KEYWORDS}

Authentication, Access control, SDN, Optical networks, Merkle signature

\section{ACM Reference format:}

Joo Yeon Cho and Thomas Szyrkowiec. 2018. Practical Authentication and Access Control for Software-Defined Networking over Optical Networks. In Proceedings of ACM SIGCOMM 2018 Workshop on Security in Softwarized Networks: Prospects and Challenges, Budapest, Hungary, August 24, 2018 (SecSoN'18), 6 pages

DOI: $10.1145 / 3229616.3229619$

\section{INTRODUCTION}

Optical networks have been widely adopted in high-bandwidth applications such as data center interconnection due to their highspeed data transmission capabilities. Modern optical networks

*Produces the permission block, and copyright information

Permission to make digital or hard copies of all or part of this work for personal or classroom use is granted without fee provided that copies are not made or distributed for profit or commercial advantage and that copies bear this notice and the full citation on the first page. Copyrights for components of this work owned by others than ACM must be honored. Abstracting with credit is permitted. To copy otherwise, or republish, to post on servers or to redistribute to lists, requires prior specific permission and/or a fee. Request permissions from permissions@acm.org.

SecSoN'18, Budapest, Hungary

(C) 2018 ACM. 978-1-4503-5912-2/18/08_..\$15.00

DOI: $10.1145 / 3229616.3229619$ become complex and handle huge amount of data, which create many difficulties for the operators to control and manage their networks. Software defined networking (SDN) provides an integrated framework to solve such difficulties in a flexible and cost-effective way.

SDN decouples the control plane from the data plane. A centralized controller maintains the state of the network and decides on the forwarding rules for the data plane. The network devices in the data plane then handle the passing traffic according to these rules, e.g., flow rules in OpenFlow. SDN offers a highly programmable infrastructure, enabling software to compute an optimal route on demand, taking constraints into account.

The SDN paradigm enables the deployment of centralized and integrated security policies, which simplifies the solution of complex network security problems. On the other hand, a malicious behavior or misuse of the control plane can compromise the proper operation of the entire network. Therefore, authentication and access control are essential primitives to ensure proper SDN operation. In Fig. 1, an SDN architecture is depicted to highlight the interfaces which critically require authentication and access control.

In the past, several Authentication, Authorization and Accounting (AAA) solutions have been proposed for SDN. AuthFlow [19] is an authentication and access control mechanism based on host credentials. AuthFlow is implemented at the data link layer with an OpenFlow (OF) controller, an authenticator, and a RADIUS server. A similar scheme is proposed in [8] for the SE (Security Enhanced) Floodlight [28]; the authors combine the existing network architecture with SDN-enabled switches, which need to be registered, authenticated and bound to hosts. In [33], the AAA management mechanisms for adoption of SDN experimentation facilities (SEFs) are introduced; the authors propose a transferable certificate-based AAA that can be implemented in any facility. OpenDaylight AAA [25] provides a security module to authenticate identities, authorize administrative access to processes and applications, and provide accounting information to log accesses to resources.

However, most known authentication and access control schemes share a common characteristic; the provers have to perform cryptographic computations such as modular exponentiation (e.g. for RSA signature) or discrete logarithm (e.g. for Diffie-Hellman key exchange). This is not a trivial task for optical layer devices since cryptographic libraries such as OpenSSL [27] or Transport-Layer Security (TLS), suitable for higher-layer security, should be installed adaptively, depending on their CPUs and operating systems.

In this paper, we propose a flexible and easily-deployable authentication and access control mechanism for SDN over optical networks. The proposed scheme is based on Merkle hash tree, in 


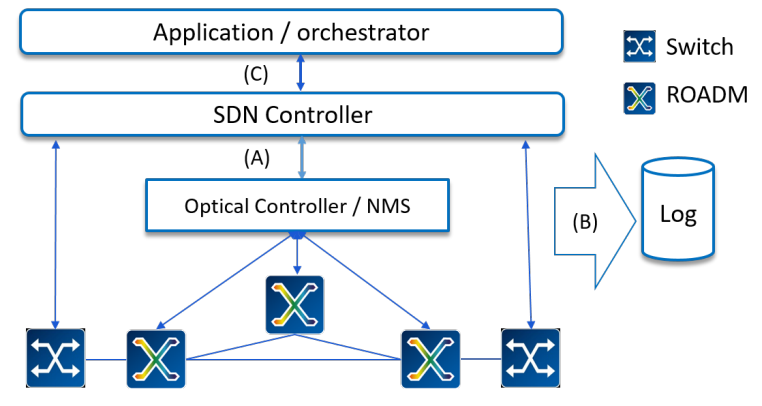

Figure 1: SDN Interfaces requiring authentication and/or access control: (A) Southbound control interface (B) Logging interface for secure recording and network forensics (C) Northbound application interface

particular, the hash-based signature and tree-based / attribute-based access control. They are easily operated on network devices without the need for computationally expensive mathematical operations. It is known that hash-based signature is resistant to any known attacks including quantum attacks [7], which allows the proposed scheme to be used for long-term security. The proposed scheme enables a fine-grained access control for orchestrators, applications, and logging in the context of SDN.

We note that hash-based signature and tree-based access control are not new, but already known mechanisms [13, 21]. Our contribution is to integrate such mechanisms, build an authentication and access control protocol for SDN, and analyze the benefits and disadvantages of our solution, compared to the standard mechanism based on conventional public-key cryptography.

For a concrete use case, we apply our solution to a SDN over optical network. The main reason is that the standard security protocol such as Transport Layer Security (TLS) [29] is often not implemented on optical devices such as optical switches, DWDMs, or ROADMs. The TLS protocol aims to allow secure communications over the transport layer or higher. For the optical layer (or layer 1), there is no such standard security protocol. The TLS protocol might be used for a secure optical communication, but the TLS software package contains many unnecessary components and the handshake protocol is not very efficient for the high speed optical transmission. The optical network is now being evolved to the SDN framework. However, due to the lack of security features on the optical devices, an authentication and access control procedure is often ignored even though a SDN controller has full functionalities to perform it. We target to provide security mechanisms that may solve this problem.

Note that our solution might be used for other scenarios. However, we are not sure that such other scenarios are always able to get benefits from our solution instead of the standard security protocol since they may have different application and environment. The situation is different when an optical SDN is considered. Optical devices composing the infrastructure of the SDN are usually based on embedded systems. A standard security protocol such as TLS can be activated when their source code and relevant cryptographic libraries such as OpenSSL are cross-compiled and installed properly. Whereas, our solution needs only cryptographic hash functions that do not need a time-consuming installation procedure. Such a hash function can be implemented in a compact way. For instance, the FIPS-202-defined SHA-3 and SHAKE functions is written in only 156 lines of code in [18]

The following sections are structured as follows: first, we identify the major security threats in SDN. As a solution, we propose a mutual authentication mechanism and an attribute-based access control mechanism. Finally, we conclude the paper.

\section{SECURITY THREATS ON OPTICAL NETWORKS IN SDN FRAMEWORK}

In this section, we identify major security threats on optical networks. We identify the following three categories for typical security threats on the optical networks.

Eavesdropping/Tapping. Eavesdropping is to attempt an unauthorized access to the carried data for the purpose of stealing data or analyzing the network traffic without breaking the connection. It is not very difficult to tap the fiber: One can tap the fiber using a commercially available clip-on coupler that can detect the leaked optical signal caused by a bend in the fiber. A more complex method is to observe the signal leaked due to crosstalk in optical switching and perform eavesdropping.

Optical disrupting. These attacks aim at degrading or completely disrupting service at the optical layer via optical link cuts, signal insertion, or signal splitting. For instance, high-power jamming, amplifier-transient or mixed-modulation attacks can cause a series of harmful effects to the co-propagating user signals inside optical fibers, amplifiers, and switches, resulting in reduced optical signal-to-noise ratio (OSNR) and degraded bit error ratio (BER) [10].

Network intrusion. In these attacks, an attacker attempts to intrude a network, access resources, and manipulate the network operation. Malicious applications and network devices may allow an attacker to introduce vulnerabilities to the system. This type of attack is critical to optical networks that are managed by an SDN controller because the attacker may try to hijack a SDN controller and control the entire network system. Hence, both an authentication and an access control mechanism are required to avoid such unauthorized access to the network.

\section{AUTHENTICATION MECHANISM}

Suppose two entities in SDN want to establish an authenticated connection. This requirement could arise at the control interface, the application interface or the logging interface, as shown in Fig. 1. A mutual authentication between two devices can be achieved by a secure communication protocol such as Transport Layer Security (TLS). A standard TLS protocol requires the public key infrastructure (PKI) based on X.509 certificates.

However, due to the evolving nature of open protocols and the technical difficulties, the TLS-based authentication is often not fully implemented nor activated in SDN devices. For example, according to OpenFlow [26], which is widely used in the major open-source SDN controllers, the use of TLS is not enforced between a switch and a controller, even though it is clear that the lack of authentication leads a path for attackers to infiltrate SDN networks, using the potential attacks described in Section 2. 


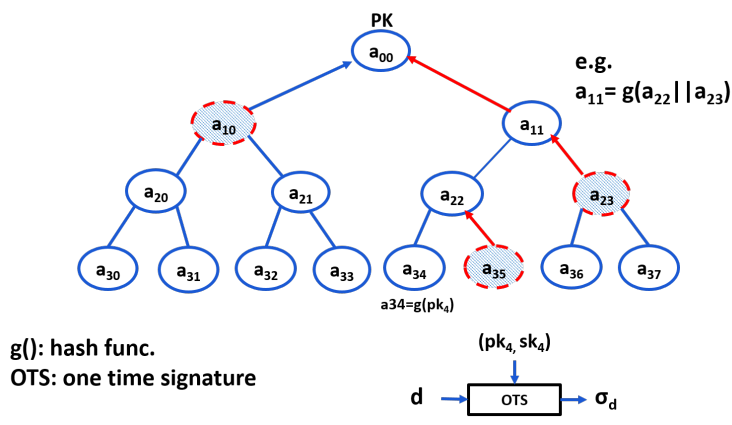

Figure 2: An example of Merkle Hash Tree with $H=3$. Dashed nodes denote the authentication path for leaf $g\left(p k_{4}\right)$. Arrows indicate the path from leaf $g\left(p k_{4}\right)$ to the root $a_{00}$.

We propose a compact and flexible authentication protocol which is based on the Merkle Signature Scheme [21]. We limit the scope of our scheme to a challenge-response authentication protocol, where we allow short challenges and use hash functions with a small output size. The security assumptions rely on those of a cryptographic hash function: pre-image resistance and collision resistance. No random oracle or number-theoretic assumptions are required.

\subsection{Hash-based Signature}

Hash-based signature (HSS) was initially proposed by Merkle in the late 1970s [21]. HSS does not rely on the conjectured hardness of mathematical problems. Instead, it is proven that it relies only on the properties of cryptographic hash functions. Hash-based signature schemes generally feature small private and public keys as well as fast signature generation and verification but large signatures and relatively slow key generation [2, 14, 20]. Unlike most other signature systems, it is known that hash-based signatures can withstand attacks using quantum computers [7].

Cryptographic hash function. A cryptographic hash function maps an input of arbitrary length to an $n$ bytes output which is computationally infeasible to invert. This feature is called the pre-image resistance. It also satisfies the collision-resistance, i.e., it is computationally infeasible to find any two distinct inputs $x$ and $x^{\prime} \operatorname{such}$ that $\operatorname{hash}(x)=\operatorname{hash}\left(x^{\prime}\right)$. A formal definition of the cryptographic hash function is given in [30].

Merkle signature scheme. Merkle signature scheme (MSS) consists of a one-time signature scheme (OTS) and a Merkle hash tree [21]. An OTS scheme can sign only one message with one key. A Merkle hash tree enables us to sign multiple messages with a single public key. An example of Merkle signature scheme is shown in Fig. 2. The leaves are the hash values of One Time Signature (OTS) public keys. Each inner node is computed as the hash of the concatenation of its two children nodes, i.e., $a_{i j}=g\left(a_{(i+1)(2 j)}|| a_{(i+1)(2 j+1)}\right)$ where $g$ is a hash function and $\|$ is a concatenation.

To sign a message $M$, a signer first computes an $n$ bytes digest $d=g(M)$. Then, the signer generates the one-time signature $\sigma_{d}$ of the digest using the $i$ th OTS key $s k_{i}$. Note that $i$ is the index chosen from $\left\{0, \ldots, 2^{H}-1\right\}$ and $H$ is the height of the tree. The $i$ th OTS key should be used only once. The signature of the message $M$ is Sign $=\left(i, \sigma_{d}, p k_{i}, A u t h_{i}\right)$, containing the index $i$, the OTS signature $\sigma_{d}$, the $i$ th OTS public key $p k_{i}$, and the authentication path $A u t h_{i}$. The authentication path $A u t h_{i}$ consists of the siblings for all nodes along the path from the $i$ th leaf to the root. In the example shown in Fig. 2, Auth $h_{i}=\left\{a_{35}, a_{23}, a_{10}\right\}$.

To verify the signature $S$ on message $M$, the verifier first validates the OTS signature on the message by $p k_{i}$. Then, a root value is computed by using the nodes in $A u t h_{i}$. If this root value matches the one given as the public key, the signature is accepted, otherwise it is rejected.

Advanced hash-based signature schemes. Since Merkle invented hash-based signatures in 1979, several variants have been proposed to improve the efficiency: XMSS (eXtended Merkle Signature Scheme) [14], XMSSMT [16], HSS (Hash-Based Signatures) [20], SPHINCS [2], and SPHINCS+ [1]. In particular, XMSS and HSS have been proposed in the Internet Engineering Task Force (IETF). We omit the detailed description of these schemes since it is straightforward to replace MSS by these schemes. Recently, Picnic [6] has been proposed, which is another promising hash-based signature, but not a variant of Merkle signature.

\subsection{Authentication Protocol}

Let us assume that a SDN controller is capable of controlling $t$ network devices. Each network device generates a hash-based public key and creates a certificate. The certificate management such as enrollment, renewal, and revocation is based on Simple Certificate Enrollment Protocol (SCEP) [12].

Enrollment. It is assumed that the enrollment of a certificate is performed in an attack-free channel. Suppose that a network device $v$ derives a public key $P K_{v}$. The certificate issuer, which can be a SDN controller itself or a trusted application, generates the following certificate $\operatorname{Cert}_{v}=\left\{P K_{v}, S I G N_{C}\right\}$, where

$$
\operatorname{SIGN}_{C}=\left(i, \operatorname{OTS}_{C}\left(P K_{v}\right), p k_{i}, A u t h_{i}\right)
$$

and $i \in\left\{0, \ldots, 2^{H_{C}}-1\right\}$. This certificate is distributed to the network device $v$, together with the public key $P K_{\text {issuer }}$. For simplicity, we assume that $P K_{\text {issuer }}=P K_{C}$.

The Merkle tree height $H$ allows to make $2^{H}$ signatures using one keypair. Since a SDN controller is usually capable of generating more signatures than network devices, we assume $H_{C} \geq H_{v}$ and $0<t \leq 2^{H_{C}}$. The security parameter $n$ and cryptographic hash function $g$ are determined on the basis of the security level required for SDN.

Authentication. Suppose a network device $v$ and a SDN controller $C$ establish a mutually authenticated connection. The proposed authentication protocol is summarized in Fig. 3. In a nutshell, they execute the following mutual authentication protocol:

(1) The network device $v$ initiates an authentication connection by sending $\mathrm{ClientHello}$ to the controller $C$.

(2) The controller $C$ generates a nonce (only used once) $r_{1}$, signs $r_{1}$ with its OTS key, and sends ServerHello and token $_{C}$ to the network device $v$. Note that token $_{C}=$ $\left\{\operatorname{Cert}_{C}, r_{1}, \operatorname{Sign}_{C}\left(i, r_{1}\right)\right\}$ for $i \in\left\{0, \ldots, 2^{H_{C}}-1\right\}$, and

$$
\operatorname{Sign}_{C}\left(i, r_{1}\right)=\left(i, \operatorname{OTS}_{C}\left(r_{1}\right), p k_{i}, \text { Auth }_{i}\right) .
$$




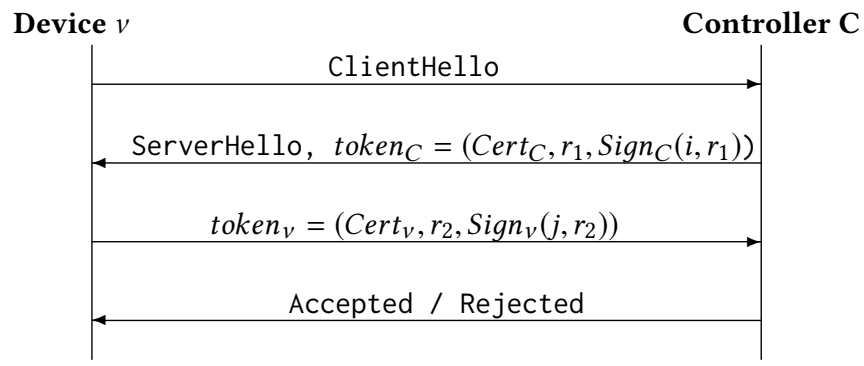

Figure 3: Hash-based authentication protocol

(3) The network device $v$ verifies the signature on $\operatorname{Cert}_{C}$ and validates $P K_{C}$. Note that $P K_{\text {issuer }}$ was initially distributed to the network device $v$ in the enrollment step. Then, the network device $v$ verifies the $\operatorname{Sign}_{C}\left(i, r_{1}\right)$ with $P K_{C}$ as described in Section 3.1.

(4) The network device $v$ generates a nonce $r_{2}$, signs $r_{2}$ with its OTS key, sends a token to the controller $C$ as follows: token $_{v}=\left\{\operatorname{Cert}_{v}, r_{2}, \operatorname{Sign}_{v}\left(j, r_{2}\right)\right\}$, where $j \in\left\{0, \ldots, 2^{H_{v}}-\right.$ $1\}$ and

$$
\operatorname{Sign}_{v}\left(j, r_{2}\right)=\left(j, \operatorname{OTS}_{v}\left(r_{2}\right), p k_{j}, A u t h_{j}\right) .
$$

(5) The controller $C$ verifies the token $_{v}$ similarly to Step (3).

\subsection{Security}

The security parameter $n$ is the output length of the hash function. Parameters with $n=32$ provide a classical security level of 256 bits and parameters with $n=64$ provide a classical security level of 512 bits. Considering attacks using quantum computers, these output sizes yield post-quantum security of 128 and 256 bits, respectively. For the $n=32$ and $n=64$ settings, the hash function $g$ can be SHA2256, SHA2-512 as defined in [22], and the SHA3/Keccak-based extendable output functions SHAKE-128, SHAKE-256 as defined in [23], respectively.

The height of the Merkle hash tree $H$ determines the number of signatures $2^{H}$. In general, hash-based signature schemes benefit when $H$ is small; the number of OTS key pairs are small, thereby fast signature generation and verification are possible.

Due to the one-time signature property, the OTS key should be used only once. To prevent OTS keys from being used twice, the OTS key pairs are numbered from 0 to $2^{H}-1$ according to the related leaf, starting from index 0 for the leftmost leaf. The OTS key contains an index that is updated with every signature generation, such that it contains the index of the next unused OTS key pair.

\section{ACCESS CONTROL MECHANISM}

The purpose of access control is to protect data, services, and network resources from unauthorized operations. In an SDN environment, the relationship between SDN controller and network devices is not static and often updated in software. Hence, the traditional identity based access control or Role-Based Access Control model (RBAC) is not effective because this model employs pre-defined identities or roles that are assigned to individual. Rather, Attribute

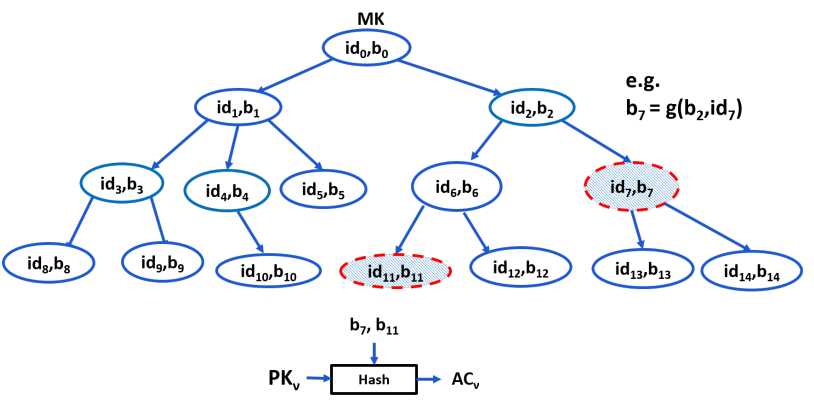

Figure 4: An example of a hash-based ABAC with $H=3$. Dashed nodes represent the associated attributes with a network device $v$. The access credential $A C_{v}$ is created by hashing the associated attributes, together with its public key $P K_{v}$.

Based Access Control (ABAC) [13] is suitable for the SDN framework. Network devices are identified by their attributes rather than predefined identities or roles.

Attributes are characteristics that define specific aspects of the entity such as a role, a location, an IP address or a validity period. ABAC evaluates available descriptive data (attributes) against stored policies to determine whether the entity is authorized to access the requested resource. Therefore, ABAC is able to provide a finegrained access control mechanism for SDN-based optical networks.

\subsection{Access Control Protocol}

The proposed $\mathrm{ABAC}$ mechanism is based on a hash tree, similar to the authentication mechanism presented in Section 3.1. The main difference is that the proposed ABAC mechanism uses a top-down hash tree. (Note that Merkle hash tree uses a bottom-up tree.) A system administrator creates a hash tree with the height $H$. The height of hash tree is determined by the structure of attributes defined in the access control policy. The nodes of the tree corresponds to network resources. Each node of the hash tree has an unique identification number $i d$. Note that all $i d s$ are public.

The system administrator generates a master key, denoted by $M K$, and assign $M K$ to the top of the tree. An attribute value of the root node is $b_{0}$ that is computed by hashing $M K$; i.e., $b_{0}=g(M K)$. From $b_{0}$, an attribute value of each node is computed by hashing the $i d$ of the node and an attribute value of the parent node. Suppose $m$ is the number of nodes in the tree. Then, for $0<i, j<m$, $b_{j}=g\left(b_{i}, i d_{j}\right)$ where the node $i$ is the parent node of the node $j$. We show an example of the hash-based ABAC mechanism in Fig. 4.

System resources are labeled with a set of attributes, based on the access control policy. Network devices, applications, or users are able to access the network resources if their credentials satisfy the attributes associated with the resources. Suppose that a network device $v$ has a $P K_{v}$, which is generated by an authentication hash tree, and has an access right on the network resources associate with $\left(b_{i_{1}}, \ldots, b_{i_{n}}\right)$. Then the access credential of the device $v$ can be created as follows:

$$
A C_{v}=g\left(P K_{v}, b_{i_{1}}, \ldots, b_{i_{n}}\right)
$$


Fig. 4 shows an example of ABAC using a hash tree. Suppose a network device $v$ has an access right associated with the attributes $b_{7}$ and $b_{11}$. Then, the device $v$ is allowed to access the nodes associated with $b_{7}$ and $b_{11}$ as well as their sub nodes such as $b_{13}$ and $b_{14}$ since $b_{13}=g\left(i d_{13}, b_{7}\right)$ and $b_{14}=g\left(i d_{14}, b_{7}\right)$. However, it cannot access the node associated with $b_{12}$, for example.

\subsection{Security}

Similarly to the authentication mechanism in Section 3, the security of proposed access control relies only on that of hash functions. Hence, the security analysis, presented in Section 3.3, can be applied to the proposed access control mechanism. We believe this is one of the advantages of the proposed mechanisms because a network operator can apply a unified method for security analysis on the authentication and access control.

\subsection{Attribute-based Encryption}

The proposed ABAC mechanism can be extensively applied to Attribute-based Encryption (ABE) [32] for SDN. ABE is an advanced encryption technique where encryption is performed with a set of descriptive attributes, so that decryption is possible only when a decryptor's attributes are matched with the one specified in the ciphertext.

ABE can be useful to handle a fine-grained encryption mechanism for SDN. The use case of ABE would be a system log. Sensitive data such as traffic log data are required to be stored in an encrypted form so that it will remain secure even if the log database is compromised. If the data is encrypted with an arbitrary number of associated attributes, a decryption access is granted if a user's attributes match the encrypted data on the access structure. Hence, the whole log data leakage by a single access can be avoided.

Here is an example. Suppose that the node $\left(i d_{6}, b_{6}\right)$ and $\left(i d_{7}, b_{7}\right)$ in Fig. 4 are associated with the log data in 2017 and 2018, respectively. Also, their sub nodes are associated with the first and second half of the year. Let us assume that it is required to audit the log data from the second half of 2017 until the end of 2018. Then, it is sufficient to provide a credential associated with only $b_{7}$ and $b_{12}$ to an auditor. With this credential, the auditor is capable to access the log data associated to $b_{12}$ and $b_{7}$ and their sub nods, $b_{13}$ and $b_{14}$, but not any other nodes. This is useful in practice for a system operator because he does not need to submit a specific period of log data to the auditor. Instead he just provides a credential associated with requested attributes and allow the auditor to access the log database.

KP-ABE vs CP-ABE. There are two approaches for AttributeBased Encryption: key-policy attribute based encryption (KP-ABE) [11] and ciphertext-policy attribute-based encryption (CP-ABE) [3]. In $\mathrm{KP}-\mathrm{ABE}$, a user can decrypt the ciphertext if and only if the access tree in his private key is satisfied by the attributes in the ciphertext. However, the encryption policy is described in the keys, so the encryptor does not have entire control over the encryption policy. Furthermore, when a new attribute is introduced, all the users must have their private keys re-issued to grant or deny access linked to the new attribute, and this process causes considerable problems in implementation.
Whereas, in the $\mathrm{CP}-\mathrm{ABE}$, ciphertexts are created with an access structure, which specifies the encryption policy, and private keys are generated according to users attributes. A user can decrypt the ciphertext if and only if his attributes in the private key satisfy the access tree specified in the ciphertext. By doing so, the encryptor holds the ultimate authority about the encryption policy. Also, the already issued private keys will never be modified unless the whole system reboots.

ABE using a hash tree. Based on the hash tree of access control that was discussed previously, ABE can be performed as follows. An encryptor decides a set of attributes which are associated with the data to be encrypted. Then, an attribute key is generated by hashing the attribute values of the associated nodes on the hash tree. Note that associated attributes are linked with not only AND gates but also with $\mathbf{O R}$ gates. For instance, suppose an encrypted file can be decrypted by a user who has both attributes $b_{4}$ and $b_{11}$ or a single attribute $b_{7}$, i.e., $\left(b_{4} \wedge b_{11}\right) \vee b_{7}$, as shown in Fig. 4 . In this case, a pair of attribute keys are created as follows: $k_{a t t 1}=g\left(b_{4}, b_{11}\right)$ and $k_{\text {att } 2}=g\left(b_{7}\right)$. The key $K$ is encrypted with this pair of attribute keys, $E\left(k_{a t t 1}, K\right)$ and $E\left(k_{a t t 2}, K\right)$ and attached to an encrypted file. Since the size of $K$ is not big, the overhead of encryption with two attribute keys is not big, either.

\section{PRACTICAL ASPECTS}

The practicability of hash-based signature schemes has been demonstrated in several papers, e.g., see $[2,4,5,9,17]$. There, the hashbased signatures have been implemented on constrained devices such as an 8-bit AVR and a 16-bit smart card at a speed comparable to RSA and ECDSA.

Suppose we choose XMSS scheme with a tree height $H=20$ and $n=32$ (e.g. SHA-2-256). This parameter set allows to generate $2^{20} \approx$ one million signatures with the security level of $2^{236}$ [15]. Then, the sizes of a public-key, a secret key, and a signature are around $2.2 \mathrm{kB}, 1.3 \mathrm{kB}$, and $2.8 \mathrm{kB}$, respectively. According to [15], a signing process, fully implemented in $C$ language, takes $3.24 \mathrm{~ms}$ on a $3.5 \mathrm{GHz}$ Intel i7 CPU platform.

We note that the size of hash-based signature and their performance are flexible, depending on the tree height and security level. A length of XMSS signature, for example, is $(4+n+($ len $+H) * n)$-byte where len is relevant to an OTS key. Hence, hash-based signature schemes would be beneficial in terms of a signature size and a signing speed if the number of network devices (hence, $H$ ) in SDN are not too big. In the standard proposal of XMSS [14], it is recommended to use $H=10,16$, or 20 with $n=32$ or 64 .

The limitation of the hash-based authentication is to allow a signature key to be used only once that comes from the characteristic of one-time signature. Recently proposed signature schemes such as SPHINCS or SPHINCS+, which are called stateless hash-based signatures, do not need to memorize the previous history of key usage, which removes such limitation. However, they require more time for signing and the size of signature is much larger.

For the easy deployment, it would be better to use a pythonbased implementation of a hash-based signature scheme, e.g. [20]. Since most of the optical devices provide a python environment due to a web-based configuration, our authentication and access control schemes can be deployed by copy-and-pasting our code, 
which is much easier than compile-and-installing cryptographic libraries on the embedded platform.

\section{CONCLUSION AND FUTURE PLAN}

In this paper, we present a hash-based authentication and an access control mechanism which are suitable for protecting a SDN-based optical network against optical security threats. The proposed mechanisms are based on Merkle hash tree, which is built using only cryptographic hash functions. Hence, the proposed mechanisms can be easily deployed on the optical network devices without computationally expensive operations.

As a next step, we will implement the proposed schemes on open source SDN framework such as ONOS [24] or Ryu [31] and apply them to optical networks. An X.509-like certificate with a hash-based signature will be generated and a python-based mutual authentication will be performed between a SDN controller and network devices. Generating a hash tree for access control can be done using the same hash functions that are used for an authentication hash tree, which reduces the code size and the attack surface. We will also compare the proposed scheme to open source SDN controllers that have implemented other authentication and access control mechanisms.

\section{ACKNOWLEDGMENTS}

This work has been performed in the framework of the CELTIC EUREKA project SENDATE-Secure-DCI (Project ID C2015/3-4), and it is partly funded by the German BMBF (Project ID 16KIS0477K).

\section{REFERENCES}

[1] Bernstein, D., Dobraunig, C., Eichlseder, M., Fluhrer, S., Gazdag, S., Hülsing, A., Kampanakis, P., Kölbl, S., Lange, T., Lauridsen, M., Mendel, F., Niederhagen, R., Rechberger, C., Rijneveld, J., and Schwabe, P. Sphincs+: Submission to the nist post-quantum project, 2017

[2] Bernstein, D., Hopwood, D., Hülsing, A., Lange, T., Niederhagen, R., Papachristodoulou, L., Schneider, M., Schwabe, P., AND WilcoX-O’Hearn, Z. Sphincs: Practical stateless hash-based signatures. In Advances in CryptologyEUROCRYPT 2015 (2015), pp. 368-397.

[3] Bethencourt, J., SAhai, A., And Waters, B. Ciphertext-policy attribute-based encryption. In Proc. IEEE Symposium on Security and Privacy (2007), pp. 321 334.

[4] Buchmann, J., DAhmen, E., AND HÜlsing, A. XMSS - a practical forward secure signature scheme based on minimal security assumptions. Cryptology ePrint Archive, Report 2011/484, 2011. https://eprint.iacr.org/2011/484.

[5] Busold, C., Buchmann, J., AND Hülsing, A. Forward secure signatures on smart cards. In Selected Areas in Cryptography, SAC 2012 (2012), vol. 7707, pp. 66-80.

[6] Chase, M., Derler, D., Goldfeder, S., Orlandi, C., Ramacher, S., Rechberger, C., Slamanig, D., and Zaverucha, G. Post-quantum zero-knowledge and signatures from symmetric-key primitives. Cryptology ePrint Archive, Report 2017/279, 2017. https://eprint.iacr.org/2017/279.

[7] Chen, L., Jordan, S., Liu, Y., Moody, D., Peralta, R., Perlner, R., and SmithTONE, D. Report on post-quantum cryptography, 2016. NISTIR 8105.

[8] Dangovas, V., AND Kuliesius, F. Sdn-driven authentication and access control system. In The International Conference on Digital Information, Networking, and Wireless Communications (DINWC2014) (2014).

[9] Eisenbarth, T., Maurich, I., AND Ye, X. Faster hash-based signatures with bounded leakage. In Selected Areas in Cryptography, SAC 2013 (2013), vol. 8282, pp. 223-243.

[10] FurdeK, M., AND SKorin-KAPOv, N. Physical-layer attacks in all-optical WDM networks. In 2011 Proceedings of the 34th International Convention MIPRO (May 2011), pp. 446-451.

[11] Goyal, V., Pandey, O., Sahai, A., and Waters, B. Attribute based encryption for fine-grained access conrol of encrypted data. In ACM conference on Computer and Communications Security (ACM CCS) (2006).

[12] Gutmann, P. Simple certificate enrollment protocol, March 2018. draft-gutmannscep-10.
[13] Hu, V., Ferraiolo, D., Kuhn, R., Schnitzer, A., Sandlin, K., Miller, R., And SCARFOnE, K. Guide to attribute based access control (ABAC) definition and considerations, 2014. NIST Special Publication (SP) 800-162.

[14] Huelsing, A., Butin, D., Gazdag, S., Rijneveld, J., And Mohaisen, A. XMSS: Extended Hash-Based Signatures. Internet-Draft draft-irtf-cfrg-xmss-hash-basedsignatures-12, Internet Engineering Task Force, Jan. 2018. Work in Progress.

[15] Hülsıng, A. Hash-based signatures. Summer School on Post-Quantum Cryptography, Eindhoven, Netherlands, June 2017. https://huelsing.files.wordpress. com/2017/06/20170619_pq_summer_school.pdf.

[16] Hülsing, A., Rausch, L., AND BuchmanN, J. Optimal parameters for $X M S S^{M T}$. Cryptology ePrint Archive, Report 2017/966, 2017. https://eprint.iacr.org/2017/ 966.

[17] Hülsing, A., Rijneveld, J., And Schwabe, P. Armed SPHINCS - computing a $41 \mathrm{~kb}$ signature in 16kb of ram. Cryptology ePrint Archive, Report 2015/1042, 2015. https://eprint.iacr.org/2015/1042.

[18] Kotewicz, M. A tiny implementation of SHA-3, SHAKE, Keccak, and sha3sum in rust, 2018. https://github.com/coruus/keccak-tiny.

[19] Mattos, D., And Duarte, O. Authflow: authentication and access control mechanism for software defined networking. Annals of Telecommunications 71, 11 (2016), 607-615.

[20] McGrew, D., Curcio, M., And Fluhrern, S. Hash-based signatures, 2018. https://tools.ietf.org/html/draft-mcgrew-hash-sigs-10.

[21] Merkle, R. A certified digital signature. In Advances in Cryptology - CRYPTO '89, 9th Annual International Cryptology Conference, Santa Barbara, California, USA, August 20-24, 1989, Proceedings (1989), pp. 218-238.

[22] NIST. Secure hash standard (SHS), 2012. FIPS 180-4

[23] NIST. SHA-3 standard: Permutation-based hash and extendable-output functions, 2015. FIPS 202.

[24] ONOS. Open network operating system, 2018. https://onosproject.org/.

[25] OpenDaylight. Authentication, authorization and accounting (AAA) services, 2004. http://docs.opendaylight.org/en/stable-nitrogen/user-guide/ authentication-and-authorization-services.html\#.

[26] OpenFlow. Openflow switch specification, 2014. Version 1.5.0, ONF TS-020

[27] OpenSSL. Cryptography and SSL/TLS toolkit, 2018. https://www.openssl.org/.

[28] Porras, P., Cheung, S., Fong, M., Skinner, K., and Yegneswaran, V. Securing the software-defined network control layer. In Proceedings of the 2015 Network and Distributed System Security Symposium (NDSS) (2015).

[29] ResCorla, E. The transport layer security (TLS) protocol version 1.3, March 2016. Internet-Draft draft-ietf-tls-tls13-12.

[30] Rogaway, P., AND Shrimpton, T. Cryptographic hash-function basics: Definitions, implications and separations for preimage resistance, second-preimage resistance, and collision resistance, 2004. Cryptology ePrint Archive, Report 2004/035.

[31] RYU. Component-based software defined networking framework, 2018. https://osrg.github.io/ryu/.

[32] Sahai, A., AND Waters, B. Fuzzy identity-based encryptionh. In Proceedings of Advances in Cryptology - EUROCRYPT 2005 (2005), vol. 3494, pp. 457 - 473.

[33] Toseef, U., ZaAlouk, A., Rothe, T., Broadbent, M., And Pentikousis, K. C-bas: Certificate-based aaa for sdn experimental facilities. In Third European Workshop on Software Defined Networks (EWSDN) (2014), pp. 91-96. 